\title{
Amino Acid Substitutions in GyrA and ParC are Associated with Fluoroquinolone Resistance in Mycoplasma bovis Isolates from Japanese Dairy Calves
}

\author{
Toyotaka SATO ${ }^{1)}$, Torahiko OKUBO ${ }^{1)}$, Masaru USUI ${ }^{1)}$, Hidetoshi HIGUCHI') and Yutaka TAMURA ${ }^{1) *}$ \\ 1) Laboratory of Food Microbiology and Food Safety, Department of Health and Environmental Sciences, School of Veterinary Medicine, \\ Rakuno Gakuen University, 582 Bunkyoudai-Midorimachi, Ebetsu, Hokkaido 069-8501, Japan \\ ${ }^{2)}$ Laboratory of Animal Health, Department of Health and Environmental Sciences, School of Veterinary Medicine, Rakuno Gakuen \\ University, Ebetsu 069-8501, Japan
}

(Received 19 November 2012/Accepted 2 March 2013/Published online in J-STAGE 15 March 2013)

ABSTRACT. We investigated the contribution of quinolone resistance-determining region (QRDR) mutations to fluoroquinolone (enrofloxacin, orbifloxacin and danofloxacin) susceptibility in 58 Mycoplasma bovis isolates from dairy cattle in Japan. Fluoroquinolone non-resistant isolates (fluoroquinolone-MICs $\leq 2 \mu \mathrm{g} / \mathrm{m} l$ ) possessed no QRDR mutations (19 isolates) or Ser83Leu in GyrA (32 isolates). Fluoroquinoloneresistant isolates (fluoroquinolone-MICs $\geq 4 \mu \mathrm{g} / \mathrm{m} l$ ) possessed Ser83Leu in GyrA and Ser81Pro in ParC (3 isolates) or Ser83Phe in GyrA and Ser80Ile in ParC (4 isolates). Laboratory-derived fluoroquinolone-resistant mutants selected from 2 isolates (possessing Ser83Leu in GyrA) had an amino acid substitution in ParC at the same position (Ser80Ile or Ser81Tyr) as fluoroquinolone-resistant isolates, suggesting a concurrent amino acid substitution in ParC (Ser80 or Ser81) is important in fluoroquinolone resistance in M. bovis isolates. KEY WORDS: calves, fluoroquinolone resistance, gyrA, Mycoplasma bovis, parC.

doi: 10.1292/jvms.12-0508; J. Vet. Med. Sci. 75(8): 1063-1065, 2013

Mycoplasma bovis ( $M b)$, a causative agent of bovine respiratory disease, causes high morbidity, and has an economic impact on the cattle industry [3]. Antimicrobials, such as fluoroquinolones, macrolides and tetracyclines, are important for treating $M b$ infections and preventing its spread in dairy calves. Fluoroquinolones are broad-spectrum antimicrobials, and enrofloxacin (ENR), orbifloxacin (ORB) and danofloxacin (DAN) have been approved for use in cattle in Japan. Fluoroquinolone minimum inhibitory concentrations (MICs) against $M b$ have previously been investigated [6, 8]. $M b$ isolated from cattle in Japan showed various ENR MICs (range $\leq 0.125-4 \mu \mathrm{g} / \mathrm{m} l$ ) [8]. Ten of eleven ENR-resistant (ENR $\mathrm{MIC} \geq 2 \mu \mathrm{g} / \mathrm{m} l$ ) $M b$ isolates derived from cattle in Israel carried 2 missense mutations within quinolone resistance-determining regions (QRDRs), causing the amino acid substitutions (AASs) Ser83Phe in GyrA and Asp84Asn in ParC [6]. Although studies have suggested that QRDR mutations contribute to elevated ENR MICs, the association between QRDR mutations and fluoroquinolone MICs has yet to be confirmed in $M b$ [6]. We investigated QRDR mutations in $M b$ isolates and in vitro fluoroquinolone-selected mutants obtained from $M b$ isolates with various ENR, ORB and DAN MICs to clarify the effect of AAS in GyrA and ParC on fluoroquinolone resistance in $M b$ isolates from dairy calves in Japan.

\footnotetext{
*Correspondence to: Tamura, Y., Laboratory of Food Microbiology and Food Safety, Department of Health and Environmental Sciences, School of Veterinary Medicine, Rakuno Gakuen University, 582 Bunkyoudai-Midorimachi, Ebetsu, Hokkaido 069-8501, Japan.

e-mail: tamuray@rakuno.ac.jp

(C)2013 The Japanese Society of Veterinary Science
}

Fifty-eight $M b$ isolates were derived from nasal swabs of asymptomatic dairy calves from 51 farms located throughout Hokkaido during 2010-2011. These isolates were provided by 7 branches of the Hokkaido Agricultural Insurance Scheme (Dounan, Iburi-Hidaka, Ishikari-Sorachi, KitamiOhotsuku, Kushiro-Nemuro, Souya-Rumoi-Kamikawa and Tokachi) and were cultured in $1 \mathrm{~m} l \mathrm{NK}$ broth (Kanto Chemical, Tokyo, Japan). After culture on modified Hayflick agar (35.5 g/l of PPLO, 15\% equine serum, 2.5\% yeast extract and $0.0024 \%$ deoxyribonucleic acid; Kanto Chemical) at $37^{\circ} \mathrm{C}$ in $5 \% \mathrm{CO}_{2}$ for 3 days to 1 week, a single colony was picked, inoculated into $1 \mathrm{ml}$ of NK broth and further cultured at $37^{\circ} \mathrm{C}$ in $5 \% \mathrm{CO}_{2}$ for 3 days. ENR, ORB and DAN MICs were determined using the agar-dilution method and modified Hayflick agar $\left(10^{4}-10^{5} \mathrm{CFU}\right.$ per plate) [2]. MIC was defined as the lowest fluoroquinolone concentration causing $>50 \%$ inhibition of growth at $37^{\circ} \mathrm{C}$ in $5 \% \mathrm{CO}_{2}$ for $72 \mathrm{hr}$ [5]. Because MIC breakpoints for $M b$ have not yet been defined, the breakpoints for ENR (susceptible, $\leq 0.25 \mu \mathrm{g} / \mathrm{m} l$; resistant, $\geq 2 \mu \mathrm{g} / \mathrm{m} l$ ), ORB and DAN (susceptible: $\leq 0.25 \mu \mathrm{g} / \mathrm{m} l$; resistant: $\geq 4 \mu \mathrm{g} / \mathrm{m} l$ ) were defined by dichotomizing bacterial populations according to these MIC distributions. QRDRs of $g y r A, \operatorname{par} C, \operatorname{par} E$ and $g y r B$ were sequenced as described previously [6]. $M b$ PG45 and the 58 isolates were stratified, based on QRDR mutation genotypes (QRDR-types). To obtain in vitro-derived fluoroquinolone-selected $M b$ mutants, we used $M b$ PG45 and voluntarily selected 2 or $4 M b$ isolates with wild type or Ser83Leu mutation in GyrA, respectively, from each QRDR-type. These strains were cultured in NK broth and centrifuged; the pellets were resuspended in $0.85 \%$ $\mathrm{NaCl}$ and were spread $\left(\sim 10^{10} \mathrm{CFU}\right)$ onto modified Hayflick agar containing ENR, DAN or ORB at 2-, 4- or 8-fold higher concentrations than the respective MICs. Cells were then cultured at $37^{\circ} \mathrm{C}$ in $5 \% \mathrm{CO}_{2}$ for 1 week. Cloning was 
Table 1. Quinolone resistance-determining region (QRDR) mutation genotypes and fluoroquinolone minimum inhibitory concentrations (MICs) in Mycoplasma bovis isolates derived from cattle in Japan

\begin{tabular}{|c|c|c|c|c|c|c|c|}
\hline \multirow{3}{*}{$\begin{array}{l}\text { QRDR-mutation } \\
\text { genotype }\end{array}$} & \multirow{3}{*}{$\begin{array}{c}\text { Number of } \\
\text { Strains }\end{array}$} & \multicolumn{3}{|c|}{ MIC: $\mu \mathrm{g} / \mathrm{m} l$ (MIC range) } & \multicolumn{3}{|c|}{ QRDR-mutations ${ }^{\text {b) }}$} \\
\hline & & \multirow{2}{*}{ ENR } & \multirow{2}{*}{ ORB } & \multirow{2}{*}{ DAN } & \multirow{2}{*}{$\frac{\text { GyrA }}{\text { S83 (tct) }}$} & \multicolumn{2}{|c|}{ ParC } \\
\hline & & & & & & S80 (agt) & S81(tct) \\
\hline \multirow{2}{*}{ Wild-type } & PG45 & 0.125 & 0.03 & 0.125 & \multirow{2}{*}{-c) } & \multirow{2}{*}{-} & \multirow{2}{*}{-} \\
\hline & 19 & $0.125^{\text {a) }}(0.06-0.25)$ & $0.06(0.03-0.25)$ & $0.125(0.125-0.25)$ & & & \\
\hline I & 32 & $0.5(0.25-1)$ & $0.5(0.25-2)$ & $0.5(0.25-2)$ & $\mathrm{L}(\mathrm{ttt})$ & - & - \\
\hline II & 3 & $4(4-8)$ & $16(8-16)$ & 16 & $\mathrm{~L}(\mathrm{ttt})$ & - & $\mathrm{P}(\mathrm{cct})$ \\
\hline III & 4 & $8(8-16)$ & $8(8-16)$ & 16 & F (tat) & I (att) & - \\
\hline
\end{tabular}

ENR-Breakpoint, $\leq 0.25 \mu \mathrm{g} / \mathrm{m} l$ as susceptible and $\geq 2 \mu \mathrm{g} / \mathrm{m} l$ as resistant; ORB- and DAN-breakpoints, $\leq 0.25 \mu \mathrm{g} / \mathrm{m} l$ as susceptible and $\geq 4 \mu \mathrm{g} / \mathrm{m} l$ as resistant. a) Values were shown as modes. b) E. coli numbering. c) -, Wild-type.

ENR, enrofloxacin; DAN, danofloxacin: F, Phe; I, Ile: L, Leu; ORB, orbifloxacin; P, Pro; S, Ser.

Table 2. Quinolone resistance-determining region (QRDR) mutation genotypes and fluoroquinolone minimum inhibitory concentrations (MICs) in Mycoplasma bovis fluoroquinolone-selected mutants derived from M .bovis isolates from cattle in Japan

\begin{tabular}{|c|c|c|c|c|c|c|c|c|c|c|c|}
\hline \multirow{3}{*}{$\begin{array}{l}\text { QRDR } \\
\text { mutation } \\
\text { genotype }\end{array}$} & \multirow{3}{*}{ Strain } & \multirow{3}{*}{$\begin{array}{l}\text { Fluoroquinolone } \\
\text { concentration } \\
(\mu \mathrm{g} / \mathrm{m} l)^{\mathrm{a})}\end{array}$} & \multicolumn{3}{|c|}{$\operatorname{MIC}(\mu \mathrm{g} / \mathrm{m} l)$} & \multicolumn{6}{|c|}{ QRDR mutations ${ }^{\mathrm{b})}$} \\
\hline & & & \multirow{2}{*}{ ENR } & \multirow{2}{*}{ ORB } & \multirow{2}{*}{ DAN } & \multicolumn{2}{|c|}{ GyrA } & \multicolumn{4}{|c|}{ ParC } \\
\hline & & & & & & G81(ggt) & $\mathrm{S} 83$ (tct) & G78 (ggt) & S80 (agt) & S81(tct) & D84 (gac) \\
\hline WT & PG45 & & 0.125 & 0.03 & 0.125 & $-c)$ & - & - & - & - & - \\
\hline IV & PG45ORB0.25-1 & ORB $(0.25)$ & 0.5 & 1 & 0.5 & $\mathrm{C}(\operatorname{tgt})$ & - & - & - & - & - \\
\hline WT & $212-1$ & & 0.25 & 0.5 & 0.5 & - & - & - & - & - & - \\
\hline I & 212-1ENR0.5-1 & $\operatorname{ENR}(0.5)$ & 1 & 2 & 1 & - & $\mathrm{L}(\mathrm{ttt})$ & - & - & - & - \\
\hline I & $132-1$ & & 0.5 & 1 & 1 & - & $\mathrm{L}(\mathrm{ttt})$ & - & - & - & - \\
\hline V & 132-1ORB2-1 & ORB (2.0) & 16 & 16 & 16 & - & $\mathrm{L}(\mathrm{ttt})$ & - & I (att) & - & - \\
\hline WT & $142-1$ & & 0.125 & 0.03 & 0.125 & - & - & - & - & - & - \\
\hline $\mathrm{V}$ & 142-1DAN1-1 & DAN (1.0) & 16 & 16 & 16 & - & $\mathrm{L}(\mathrm{ttt})$ & - & I (att) & - & - \\
\hline I & $235-1$ & & 0.5 & 2 & 2 & - & $\mathrm{L}(\mathrm{ttt})$ & - & - & - & - \\
\hline VI & 235-1ENR1-1 & ENR (1.0) & 8 & 16 & 16 & - & $\mathrm{L}(\mathrm{ttt})$ & - & - & $\mathrm{Y}$ (tat) & - \\
\hline I & $254-1$ & & 0.5 & 2 & 1 & - & $\mathrm{L}(\mathrm{ttt})$ & - & - & - & - \\
\hline VII & 254-1ENR1-1 & ENR (1.0) & 8 & 8 & 8 & - & $\mathrm{L}(\mathrm{ttt})$ & $\mathrm{L}(\operatorname{tgt})$ & - & - & - \\
\hline I & $251-1$ & & 0.5 & 2 & 1 & - & $\mathrm{L}(\mathrm{ttt})$ & - & - & - & - \\
\hline VIII & 251-1ENR1-1 & ENR (1.0) & 4 & 8 & 4 & - & $\mathrm{L}(\mathrm{ttt})$ & - & - & - & Y (tat) \\
\hline
\end{tabular}

ENR-breakpoint: $\leq 0.25 \mu \mathrm{g} / \mathrm{m} l$ as susceptible and $\geq 2 \mu \mathrm{g} / \mathrm{m} l$ as resistant; ORB- and DAN-breakpoints: $\leq 0.25 \mu \mathrm{g} / \mathrm{m} l$ as susceptible and $\geq 4 \mu \mathrm{g} / \mathrm{m} l$ as resistant. a) Fluoroquinolone concentration used in agar selection. b) E. coli numbering. c) -, Wild-type.

ENR, enrofloxacin; C, Cys; D, Asp; DAN, danofloxacin: G, Gly; I, Ile; L, Leu; ORB, orbifloxacin; S, Ser; Y, Tyr.

performed at least twice using fluoroquinolone-containing agar. Mb PG45 was used as the control for susceptibility and detection of QRDR mutations (described in terms of Escherichia coli numbering).

QRDR-types and fluoroquinolone MICs of $\mathrm{Mb}$ isolates are shown in Table 1. Nineteen (32.7\%) isolates (wild type) were susceptible, and $32(55.2 \%)$ (Type I: Ser83Leu in GyrA) were susceptible or intermediate to fluoroquinolones. Modes of fluoroquinolone MICs from Type I isolates were 4to 8 -fold higher than those of the wild type. Seven (12.1\%) isolates were resistant to fluoroquinolones; these possessed AASs in GyrA and ParC (Type II: Ser83Leu in GyrA and Ser81Pro in ParC; Type III: Ser83Phe in GyrA and Ser80Ile in ParC). All these QRDR types were different from those in Israel [6].

A fluoroquinolone-selected mutant derived from a wildtype isolate had the same AAS as Type I isolates, i.e., Ser83Leu in GyrA (Table 2). This mutant showed elevated fluoroquinolone MICs, but did not exceed resistance break- points. Fluoroquinolone-selected mutants derived from Type I isolates had an additional AAS at the same position in ParC (Type V: Ser83Leu in GyrA and Ser80Ile in ParC; Type VI: Ser83Leu in GyrA and Ser81Tyr in ParC) as Type II and III isolates, and fluoroquinolone MICs for these mutants exceeded resistance breakpoints. These findings suggest that an AAS at Ser83 in GyrA is sufficient to decrease fluoroquinolone MICs, but concurrently, an AAS at Ser80 or Ser81 in ParC plays an important role in fluoroquinolone resistance in field-derived $M b$ isolates.

QRDR-types IV-VIII were detected in vitro, but not in field isolates (Table 2), possibly because of the effect of some QRDR mutations on bacterial colonization in the host [4]. 142-1DAN1-1 (Type V) demonstrated simultaneous AASs in GyrA and ParC; this phenomenon has been shown in M. gallisepticum [7], suggesting these were likely to occur during spontaneous exposure of DAN present in the supplemented agar used for selection and cloning of a 142-1 derived mutant. QRDR-types of 4 fluoroquinolone-selected 
mutants derived from different Type I isolates varied: $1 \mathrm{mu}-$ tant selected from ORB-supplemented agar was Type V, and 3 mutants selected from agars containing the same ENR concentration were Types VI-VIII. QRDR mutations occurred heterogeneously in other Mycoplasma strains from the same isolate, independent of fluoroquinolone agents or fluoroquinolone concentrations used for selecting fluoroquinoloneselected mutants in vitro [1,7]. Although these data suggest that $M b$ has the same fluoroquinolone resistance mechanism attributed to heterogeneous AASs in GyrA and ParC as other Mycoplasma species, we do not have experimental evidence to show this in $M b$ because we did not use multiple fluoroquinolone agents or fluoroquinolone concentrations for a parent strain. These results should be further investigated in future studies.

ACKNOWLEDGMENTS. This study was supported by a grant from a program for developing a supporting system for upgrading education and research from the Japanese Ministry of Education, Culture, Sports, Science and Technology and a grant from the Japanese Ministry of Agriculture, Forestry and Fisheries.

\section{REFERENCES}

1. Bébéar, C. M., Renaudin, H., Charron, A., Bové, J. M., Bébéar, C. and Renaudin, J. 1998. Alterations in topoisomerase IV and DNA gyrase in quinolone-resistant mutants of Mycoplasma hominis obtained in vitro. Antimicrob. Agents Chemother. 42:
2304-2311. [Medline]

2. Clinical and Laboratory Standards Institute. 2008. Performance standards for antimicrobial disk and dilution antimicrobial susceptibility tests for bacteria isolated from animals. Approved standard, 3rd ed. In: CLSI document M31-A3, CLSI, Wayne.

3. Gagea, M. I., Bateman, K. G., van Dreumel, V., McEwen, B. J., Carman, S., Archambault, M., Shanahan, R. A. and Caswell, J. L. 2006. Diseases and pathogens associated with mortality in Ontario beef feedlots. J. Vet. Diagn. Invest. 18: 18-28. [Medline] [CrossRef]

4. Giraud, E., Cloeckaert, A., Baucheron, S., Mouline, C. and Chaslus-Dancla, E. 2003. Fitness cost of fluoroquinolone resistance in Salmonella enterica serovar Typhimurium. J. Med. Microbiol. 52: 697-703. [Medline] [CrossRef]

5. Hannan, P. C. T. 2000. Guidelines and recommendations for antimicrobial minimum inhibitory concentration (MIC) testing against veterinary Mycoplasma species. Vet. Res. 31: 373-395. [Medline] [CrossRef]

6. Lysnyansky, I., Mikula, I., Gerchman, I. and Levisohn, S. 2009. Rapid detection of a point mutation in the parC gene associated with decreased susceptibility to fluoroquinolones in Mycoplasma bovis. Antimicrob. Agents Chemother. 53: 4911-4914. [Medline] [CrossRef]

7. Reinhardt, A. K., Kempf, I., Kobisch, M. and Gautier-Bouchardon, A. V. 2002. Fluoroquinolone resistance in Mycoplasma gallisepticum: DNA gyrase as primary target of enrofloxacin and impact of mutations in topoisomerases on resistance level. J. Antimicrob. Chemother. 50: 589-592. [Medline] [CrossRef]

8. Uemura, R., Sueyoshi, M. and Nagatomo, H. 2010. Antimicrobial susceptibilities of four species of Mycoplasma isolated in 2008 and 2009 from cattle in Japan. J. Vet. Med. Sci. 72: 1661-1663. [Medline] [CrossRef] 